\title{
A NOTE ON WEIGHTED SOBOLEV SPACES RELATED TO WEAKLY AND STRONGLY DEGENERATE DIFFERENTIAL OPERATORS
}

\author{
Peter I. Kogut, Olha P. Kupenko ${ }^{\dagger \dagger}$ Guenter Leugering§ Yue Wang
}

\begin{abstract}
In this paper we discuss some issues related to Poincaré's inequality for a special class of weighted Sobolev spaces. A common feature of these spaces is that they can be naturally associated with differential operators with variable diffusion coefficients that are not uniformly elliptic. We give a classification of these spaces in the 1-D case bases on a measure of degeneracy of the corresponding weight coefficient and study their key properties.
\end{abstract}

Key words: Degenerate equation, weighted Sobolev spaces, Poincaré's inequality..

2010 Mathematics Subject Classification: 46E35, 26D10, 35A23, 35J70.

\section{Communicated by Prof. S. Avdonin}

\section{Introduction}

In this paper we discuss some issues related to Poincaré's inequality for a special class of weighted Sobolev spaces. A common feature of these spaces is that they can be naturally associated with differential operators with variable diffusion coefficients that are not uniformly elliptic, even though they are in general uniformly elliptic in compact subsets of the domain, even though they are in general uniformly elliptic in compact subsets of the space domain, provided that these subsets are at a positive distance from the the so-called zone of degeneracy. This degeneracy zone may occur either on a part of the boundary or on a sub-manifold of the space domain.

Some aspects of this problem with a degeneration at the boundary point $x^{0}=0$ of 1-D domain $\Omega=(0,1)$ have been recently considered by Alabau-Boussouira, Cannarsa, and Leugering in [1]. In particular, if $a \in C([0,1]) \cap C^{1}((0,1])$ is a given weight coefficient with properties

$$
a(x)>0, \quad \forall x \in(0,1] \text { and } a(0)=0,
$$

\footnotetext{
*Department of Differential Equations, Oles Honchar Dnipro National University, 72, Gagarin av., Dnipro, 49010, Ukraine, p.kogut@i.ua

${ }^{\dagger}$ Department of System Analysis and Control, National Mining University, 19, Yavornitskii av., 49005 Dnipro

${ }^{\ddagger}$ Institute of Applied System Analysis, National Academy of Sciences and Ministry of Education and Science of Ukraine, 37/35, Peremogy av., IASA, 03056 Kyiv, Ukraine, kupenko.olga@gmail.com

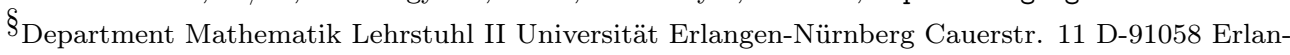
gen, Germany (guenter.leugering@fau.de)

बDepartment of Mathematics, Friedrich-Alenxander University, Erlangen-Nurmberg, Cauerstrasse 11, 91058 Erlangen, Germany, yue.wang@fau.de

(C) P. I. Kogut, O. P. Kupenko, G. Leugering, Y. Wang, 2019.
} 
then the authors in [1] propose to measure the degree of degeneracy of the function $a(\cdot)$ at $x=0$ by the parameter $\mu_{a}$ which is defined as

$$
\mu_{a}:=\sup _{0<x \leq 1} \frac{x\left|a^{\prime}(x)\right|}{a(x)} .
$$

Moreover, they propose to use the parameter $\mu_{a}$ as a main feature for the classification of the weight functions $a: \Omega \rightarrow \mathbb{R}$. They say that a function $a(\cdot)$ has a weak degeneration at $x^{0}=0$ if $\mu_{a} \in[0,1)$, and this function is strongly degenerate if $\mu_{a}>1$. It can be shown that any weakly degenerate functions $a(\cdot)$ (i.e., $0 \leq \mu_{a}<1$ ) belongs to the class of Muckenhoupt weights $A_{2}(\Omega)$, that is, $w: \Omega \rightarrow \mathbb{R}_{+}$belongs to $A_{2}(\Omega)$ if

$$
\left(\frac{1}{|B|} \int_{B} w d x\right)\left(\frac{1}{|B|} \int_{B} w^{-1} d x\right) \leq C<+\infty, \quad \forall B \subseteq \Omega,
$$

and this case of degeneration has received a lot of attention in the literature (see, for instance, $[4,6,9-12,15,16])$. At the same time, if $\mu_{a}>1$, then $a(\cdot) \notin A_{2}$ and in this case we can expect to have many new effects related to the solvability issues of the corresponding boundary value problems and their properties.

It is worth noting that such issues as controllability and observability of the corresponding degenerate systems are also closely related to the parameter $\mu_{a}$. In particular, it has been shown in [1] for degenerate wave equations of the form

$$
u_{t t}-\left(a(x) u_{x}\right)_{x}=0 \quad \text { in }(0, \infty) \times(0,1)
$$

that their observability and boundary controllability no longer hold true if $\mu_{a} \geq 2$. The same conclusion can be done for the parabolic case (see, for instance, $[2,3$, $7,13])$. So, the authors in [1] provide analysis of the above mentioned properties assuming that $\mu_{a}<2$ and for that they make use of the following weighted Sobolev spaces

$$
H_{a}^{1}(\Omega)=\left\{\begin{array}{l|l}
u \in L^{2}(\Omega) & \begin{array}{c}
u \text { is locally absolutely continuous in }(0,1], \\
\sqrt{a} u_{x} \in L^{2}(\Omega)
\end{array}
\end{array}\right\}
$$

with norm

$$
\|u\|_{H_{a}^{1}(\Omega)}=\left(\int_{\Omega}\left[a(x)\left|u^{\prime}(x)\right|^{2}+|u(x)|^{2}\right] d x\right)^{\frac{1}{2}}, \quad \forall u \in H_{a}^{1}(\Omega) .
$$

and

$$
H_{a, 0}^{1}(\Omega):=\left\{u \in H_{a}^{1}(\Omega): u(1)=0\right\} .
$$

It is well known that the loss of uniform ellipticity for operators like $A u=$ $-\left(a(x) u_{x}\right)_{x}$ raises new questions related to the well-posedness of the corresponding evolution equations in suitable functional spaces as well as new estimates for the underlying elliptic equations. With that in mind, the authors in [1] have shown 
that the Poincaré's inequality for elements of weighted Sobolev space $H_{a, 0}^{1}(\Omega)$ can be established not only in the case of weakly degenerate weight function $a(\cdot)$, but also if $\mu_{a}<2$. In particular, if $\mu_{a}<2$ it has been shown in [1] that

$$
\|u\|_{L^{2}(\Omega)} \leq \frac{1}{a(1)} \min \left\{4, \frac{1}{2-\mu_{a}}\right\}\|u\|_{H_{a}^{1}(\Omega)}, \quad \forall u \in H_{a, 0}^{1}(\Omega) .
$$

At the same time, in problems involving cloaking which, obviously, is incompatible with observability, the degeneracy of the coefficients is quadratic [8], and, hence, in this case we have $\mu_{a}=2$. Therefore, the purpose of this paper is to study the issues related to the weighted Sobolev space $H_{a, 0}^{1}(\Omega)$ provided the degeneracy zone of the weight function $a(\cdot)$ is an interior point $x^{0}$ of the domain $\Omega$ and the measure of degeneracy at this point can be equal to 2 or larger than 2 . As a sub-product of our analysis, we show that the classification of degeneracy measure of function $a(\cdot)$ essentially depends on the properties of its derivative $(\sqrt{a(x)})_{x}$. In particular, for the weight functions $a \in C([0,1]) \cap C^{1}((0,1])$ with properties (1.1), the following assertions hold true

$$
\begin{gathered}
\text { If }(\sqrt{a})_{x}^{-1} \in L^{\infty}(\Omega), \text { then } \mu_{a} \in[0,2), \\
\text { If }(\sqrt{a(x)})_{x}=\text { const in } \Omega, \text { then } \mu_{a}=2,
\end{gathered}
$$

If $(\sqrt{a})_{x} \in L^{\infty}(\Omega)$ and $(\sqrt{a(x)})_{x} \neq$ const in $\Omega$, then $\mu_{a}>2$.

The paper is organized as follows. In Section 2, we introduce our notations, define the new degeneracy parameters $A_{a, i}$ and $\mu_{a, i}, i=1,2$, and derive some auxiliary inequalities for the weight function $a: \bar{\Omega} \rightarrow \mathbb{R}$. In Section 3 , we prove the Poincaré's type inequalities for functions in weighted Sobolev space $H_{a, 0}^{1}(\Omega)$ in the case of weakly degenerate weight functions $a(\cdot)$. In particular, we show that in this case Poincare's inequality (1.2) can be extended to the following one

$$
\|u\|_{L^{2}(\Omega)} \leq\left[C_{1}+C_{2}\right]\|u\|_{H_{a}^{1}(\Omega)}, \quad \forall u \in H_{a, 0}^{1}(\Omega),
$$

where $H_{a, 0}^{1}(\Omega)=\left\{u \in L^{2}(\Omega): \sqrt{a} u_{x} \in L^{2}(\Omega), u(0)=0\right\}, a\left(x^{0}\right)=0, a(x)>0$ for all $x \in[0,1] \backslash\left\{x^{0}\right\}, x^{0} \in(0,1)$,

$$
\begin{aligned}
& C_{1}=\frac{1}{\sqrt{a(0)}} \min \left\{\frac{1}{\sqrt{\left[2-\max \left\{\mu_{1, a}, 2 A_{1, a}\right\}\right]}}, 2\right\}, \\
& C_{2}=\frac{1}{\sqrt{a(1)}}\left[\min \left\{\frac{1}{\sqrt{\left[2-\max \left\{\mu_{2, a}, 2 A_{2, a}\right\}\right]}}, 2\right\}+C_{S o b} \sqrt{1+a^{2}(1)}\right],
\end{aligned}
$$

and parameters $A_{i, a}$ and $\mu_{i, a}, i=1,2$, are given by relations (2.2), (2.3), and (2.4), and satisfy conditions (3.4). We also show in this section that if $(\sqrt{a})_{x}^{-1} \in L^{\infty}(\Omega)$ then $\max \left\{\mu_{i, a}, 2 A_{i, a}\right\}<2$ and, hence, Poincaré's inequality (1.3) remains valid. 
In Section 4, we proceed in the study of some key results for functions in weighted Sobolev space $H_{a, 0}^{1}(\Omega)$ provided the weight function $a(\cdot)$ has a large measure of degeneracy at some interior point $x^{0} \in(0,1)$. In other words, our key assumption in this section is $(\sqrt{a})_{x} \in L^{\infty}(\Omega)$. In this case, we show that the weighted space $H_{a, 0}^{1}(\Omega)$ is isomorphic to the following one

$$
V_{a, 0}^{1}(\Omega)=\left\{u \in L^{2}(\Omega): u\left(x^{0}\right)=0,(\sqrt{a} u)_{x} \in L^{2}(\Omega)\right\} .
$$

As a result, we derive another type of Poincaré's inequality for elements of $H_{a, 0}^{1}(\Omega)$. Namely, we establish the following relations (see Theorem 4.2)

$$
\|\sqrt{a} u\|_{L^{2}(\Omega)} \leq 2 \sqrt{2}\left(1+\left\|(\sqrt{a})_{x}\right\|_{L^{\infty}(\Omega)}\right)^{\frac{1}{2}}\|u\|_{H_{a}^{1}(\Omega)}, \quad \text { if } \quad(\sqrt{a})_{x} \in L^{\infty}(\Omega)
$$

and

$$
\|u\|_{L^{2}(\Omega)} \leq \frac{2 \sqrt{1+C_{*}}}{C_{*}}\|u\|_{H_{a}^{1}(\Omega)}, \quad \forall u \in H_{a, 0}^{1}(\Omega), \quad \text { if } \quad(\sqrt{a(x)})_{x}=\text { const in } \Omega .
$$

Moreover, in this case we can not guarantee that elements of the space $H_{a, 0}^{1}(\Omega)$ are continuous functions in $\Omega$ or even integrable over this domain. Instead we can assert that the following implication holds true: If $u \in H_{a, 0}^{1}(\Omega)$ and $(\sqrt{a})_{x} \in$ $L^{\infty}(\Omega)$, then $\sqrt{a(x)} u(x)$ is an absolutely continuous function in $\bar{\Omega}=[0,1]$.

\section{Assumptions and Preliminaries}

Let $x^{0} \in[0,1]$ be a given point. We set

$$
\begin{gathered}
\Omega_{1}=\left\{\begin{array}{ll}
\left(0, x^{0}\right), & \text { if } x^{0}>0, \\
\emptyset, & \text { if } x^{0}=0,
\end{array}, \quad \Omega_{2}=\left\{\begin{array}{ll}
\left(x^{0}, 1\right), & \text { if } x^{0}<1, \\
\emptyset, & \text { if } x^{0}=1,
\end{array},\right.\right. \\
\Omega=(0,1), \quad \text { and } \quad \Omega_{0}=\Omega \backslash\left\{x^{0}\right\}=\Omega_{1} \cup \Omega_{2} .
\end{gathered}
$$

We denote by $C_{0}^{\infty}(\mathbb{R})$ the locally convex space of all infinitely differentiable functions with compact support. Following the standard way, we define the Banach space $W_{0}^{1,2}(\Omega ; 0)$ as the closure of $C_{0}^{\infty}(\mathbb{R} ; 0)=\left\{\varphi \in C_{0}^{\infty}(\mathbb{R}): \varphi(0)=0\right\}$ with respect to the norm

$$
\|y\|_{W_{0}^{1,2}(\Omega ; 0)}=\left(\int_{\Omega}|\nabla y|^{2} d x\right)^{1 / 2} .
$$

We also set $C^{k, \alpha}(\Omega)$ for the Hölder space of those functions on $\Omega$ having continuous derivatives up to order $k$ and such that the $k$ th derivative is Hölder continuous with exponent $\alpha \in(0,1]$. It is well known that $C^{k, \alpha}(\bar{\Omega})$ is a Banach space with respect to the norm

$$
\|f\|_{C^{k, \alpha}(\bar{\Omega})}=\max _{0 \leq i \leq k} \sup _{x \in \Omega}\left|f^{(i)}(x)\right|+\sup _{x \neq y \in \Omega} \frac{\left|f^{(k)}(x)-f^{(k)}(y)\right|}{|x-y|^{\alpha}}
$$

and the embedding $C^{k, \alpha}(\bar{\Omega}) \hookrightarrow C^{k, 0}(\bar{\Omega})$ is compact.

Let $a: \bar{\Omega} \rightarrow \mathbb{R}$ be a given function with properties 
(i) $a\left(x^{0}\right)=0$ and $a(x)>0$ for all $x \in \bar{\Omega} \backslash\left\{x^{0}\right\}$;

(ii) $a \in C(\bar{\Omega}) \cap C_{l o c}^{1}\left(\Omega_{0}\right)$.

In what follows, we associate with the function $a: \bar{\Omega} \rightarrow \mathbb{R}$ the following degenerate elliptic operator

$$
A(y)=-\left(a(x) y_{x}\right)_{x}
$$

Let $G_{i}: \Omega \rightarrow[0, \infty), i=1,2$, be non-decreasing continuous functions such that $G_{i}(0)=0$ and

$$
\begin{aligned}
& A_{1, a}:=\sup _{x \in \Omega_{1}} \frac{G_{1}\left(x^{0}-x\right)\left|(\sqrt{a(x)})_{x}\right|}{\sqrt{a(x)}}=\sup _{x \in \Omega_{1}} \frac{G_{1}\left(x^{0}-x\right)\left|a^{\prime}(x)\right|}{2 a(x)}<+\infty, \\
& A_{2, a}:=\sup _{x \in \Omega_{2}} \frac{G_{2}\left(x-x^{0}\right)\left|(\sqrt{a(x)})_{x}\right|}{\sqrt{a(x)}}=\sup _{x \in \Omega_{2}} \frac{G_{2}\left(x-x^{0}\right)\left|a^{\prime}(x)\right|}{2 a(x)}<+\infty .
\end{aligned}
$$

By analogy with [1-3], we also set

$$
\mu_{1, a}:=\sup _{x \in \Omega_{1}} \frac{\left(x^{0}-x\right)\left|a^{\prime}(x)\right|}{a(x)}, \quad \mu_{2, a}:=\sup _{x \in \Omega_{2}} \frac{\left(x-x^{0}\right)\left|a^{\prime}(x)\right|}{a(x)} .
$$

Example 2.1. As an example of function $a: \bar{\Omega} \rightarrow \mathbb{R}_{+}$with the above indicated properties (i)-(ii), we can consider the following one (see $[5,14])$.

$$
a(x)=\left\{\begin{array}{ll}
\left(\frac{1}{2}-x\right)^{2 p_{1}}, & \text { if } x \in\left[0, \frac{1}{2}\right] \\
\left(x-\frac{1}{2}\right)^{2 p_{2}}, & \text { if } x \in\left(\frac{1}{2}, 1\right],
\end{array} \quad \text { with } p_{1}, p_{2}>0 .\right.
$$

Here, $x^{0}=\frac{1}{2}$. It is easy to check that, in this case, properties (i)-(ii) hold true. Moreover, setting

$G_{1}(x)=k_{1} x \quad$ and $\quad G_{2}(x)=k_{2} x, \quad$ where $k_{1}, k_{2}$ are some positive constants, we see that

$$
A_{1, a}=\frac{k_{1}}{2} \mu_{1, a}=k_{1} p_{1} \quad \text { and } \quad A_{2, a}=\frac{k_{2}}{2} \mu_{2, a}=k_{2} p_{2} .
$$

In addition, we have the following properties

$$
\begin{gathered}
(\sqrt{a})_{x} \in L^{\infty}(\Omega) \text { if } p_{1} \text { and } p_{2} \text { are greater than } 1, \\
\frac{1}{a} \in L^{1}(\Omega) \quad \text { provided } 0<p_{1}, p_{2}<\frac{1}{2} .
\end{gathered}
$$

Another example of a weight function $a:[0,1] \rightarrow \mathbb{R}_{+}$with $x^{0}=\frac{1}{2}$ can be described as follows:

$$
a(x):=\left\{\begin{array}{ll}
\exp \left(-\frac{2}{\left|x-\frac{1}{2}\right|^{p}}\right) & \text { if } x \in \Omega_{0}, \\
0 & \text { if } x=\frac{1}{2} .
\end{array} \quad \text { with some } p>0 .\right.
$$


After some calculus, we arrive at

$$
\begin{gathered}
\frac{(\sqrt{a})_{x}}{\sqrt{a}}=p \operatorname{sign}\left(x-\frac{1}{2}\right)\left|x-\frac{1}{2}\right|^{-p-1}, \\
\mu_{1, a}=\mu_{2, a}=+\infty
\end{gathered}
$$

whereas

$$
A_{1, a}=A_{2, a}=k p
$$

with $G_{1}(x)=G_{2}(x)=k\left|x-\frac{1}{2}\right|^{p+1}$. It is clear that, in this case, we have

$$
(\sqrt{a})_{x} \in L^{\infty}(\Omega) \quad \text { and } \quad \frac{1}{a}=\exp \left(\frac{2}{\left|x-\frac{1}{2}\right|^{p}}\right) \notin L^{1}(\Omega) \quad \forall p>0 .
$$

Before proceeding further, we list below some simple properties of function $a: \bar{\Omega} \rightarrow \mathbb{R}$ related to the given characteristics $A_{i, a}$ and $\mu_{i, a}$.

Lemma 2.1. Let $a: \bar{\Omega} \rightarrow \mathbb{R}$ be a given function with properties (i)-(ii). Let $A_{i, a}$ and $\mu_{i, a}, i=1,2$, be the values given by relations (2.2), (2.3), and (2.4). Then

$$
\begin{array}{ll}
a(x) \geq a(0)\left(x^{0}-x\right)^{\max \left\{\mu_{1, a}, 2 A_{1, a}\right\}} & \forall x \in\left[0, x^{0}\right], \\
a(x) \geq a(1)\left(x-x^{0}\right)^{\max \left\{\mu_{2, a}, 2 A_{2, a}\right\}} & \forall x \in\left[x^{0}, 1\right] .
\end{array}
$$

Proof. Since the function $G_{i}: \Omega \rightarrow[0, \infty), i=1,2$, are unknown a priori, we begin with the case when $G_{i}(s) \geq s$ for all $s \in \Omega$. Then the following relations

$$
\begin{gathered}
A_{1, a}=\sup _{x \in \Omega_{1}} \frac{G_{1}\left(x^{0}-x\right)\left|a^{\prime}(x)\right|}{2 a(x)} \geq \sup _{x \in \Omega_{1}} \frac{\left(x^{0}-x\right)\left|a^{\prime}(x)\right|}{2 a(x)}=\frac{1}{2} \mu_{1, a}, \\
A_{2, a}=\sup _{x \in \Omega_{2}} \frac{G_{2}\left(x-x^{0}\right)\left|a^{\prime}(x)\right|}{2 a(x)} \geq \sup _{x \in \Omega_{2}} \frac{\left(x-x^{0}\right)\left|a^{\prime}(x)\right|}{2 a(x)}=\frac{1}{2} \mu_{2, a} .
\end{gathered}
$$

are obvious. Therefore, making use of representation (2.4), we get

$$
\left(x^{0}-x\right) a^{\prime}(x) \stackrel{\text { by }(2.4)}{\geq}-\mu_{1, a} a(x) \stackrel{\text { by }}{\geq}{ }^{(2.9)}-2 A_{1, a} a(x), \quad \forall x \in \Omega_{1} .
$$

Integrating this inequality over $[0, x]$,

$$
\int_{0}^{x} \frac{a^{\prime}}{a} d s \geq-2 A_{1, a} \int_{0}^{x} \frac{1}{x^{0}-s} d s, \quad \forall x \in\left[0, x^{0}\right),
$$

we obtain

$$
a(x) \geq a(0)\left(x^{0}-x\right)^{2 A_{1, a}}, \quad \forall x \in\left[0, x^{0}\right] .
$$

Arguing in a similar manner, we have

$$
\left(x-x^{0}\right) a^{\prime}(x) \stackrel{\text { by }(2.10)}{\leq} G_{2}\left(x-x^{0}\right) a^{\prime}(x) \stackrel{\text { by }}{\leq} 2 A_{2, a} a(x), \quad \forall x \in\left[x^{0}, 1\right] .
$$


Therefore,

$$
\int_{x}^{1} \frac{a^{\prime}}{a} d s \leq 2 A_{2, a} \int_{x}^{1} \frac{1}{s-x^{0}} d s, \quad \forall x \in\left(x^{0}, 1\right]
$$

and, hence,

$$
a(x) \geq a(1)\left(x-x^{0}\right)^{2 A_{2, a}}, \quad \forall x \in\left[x^{0}, 1\right] .
$$

It remains to consider the second case: $G_{i}(s) \leq s$ for all $s \in \Omega$. Then

$$
\begin{gathered}
-G_{1}\left(x^{0}-x\right) a^{\prime}(x) \geq-\left(x^{0}-x\right) a^{\prime}(x)=-\frac{\left(x^{0}-x\right) a^{\prime}(x)}{a(x)} a(x) \\
\quad \text { by } \stackrel{(2.4)}{\geq}-\mu_{1, a} a(x), \quad \forall x \in\left[0, x^{0}\right) .
\end{gathered}
$$

From this, after integration over $[0, x]$, we deduce

$$
a(x) \geq a(0)\left(x^{0}-x\right)^{\mu_{1, a}}, \quad \forall x \in\left[0, x^{0}\right]
$$

Arguing in a similar manner, we have

$$
\begin{aligned}
G_{2}\left(x-x^{0}\right) a^{\prime}(x) & \leq\left(x-x^{0}\right) a^{\prime}(x) \leq a(x) \sup _{x \in \Omega_{2}} \frac{\left(x-x^{0}\right)\left|a^{\prime}(x)\right|}{a(x)} \\
& =\mu_{2, a} a(x), \quad \forall x \in\left(x^{0}, 1\right] .
\end{aligned}
$$

As a result, by integration over $\left[x^{0}, 1\right]$,

$$
a(x) \geq a(1)\left(x-x^{0}\right)^{\mu_{2, a}}, \quad \forall x \in\left[x^{0}, 1\right] .
$$

Thus, to conclude the proof, it remains to combine the inequalities $(2.11)-(2.12)$ and (2.13)-(2.14).

\section{Poincaré Inequality for a Weighted Sobolev Space}

We now introduce some weighted Sobolev spaces that are naturally associated with functions $a: \bar{\Omega} \rightarrow \mathbb{R}$ satisfying properties (i)-(ii) and with degenerate elliptic operators like $(2.1)$ (see, for instance, $[1,13])$. We denote by $H_{a}^{1}(\Omega)$ the following space of all functions $u \in L^{2}(\Omega)$ such that

$$
H_{a}^{1}(\Omega)=\left\{u \in L^{2}(\Omega): \sqrt{a} u_{x} \in L^{2}(\Omega)\right\} .
$$

It is easy to see that $H_{a}^{1}(\Omega)$ is a Hilbert space with the scalar product

$$
\langle u, v\rangle_{H_{a}^{1}(\Omega)}=\int_{\Omega}\left[a(x) u^{\prime}(x) v^{\prime}(x)+u(x) v(x)\right] d x, \quad \forall u, v \in H_{a}^{1}(\Omega)
$$

and associated norm

$$
\|u\|_{H_{a}^{1}(\Omega)}=\left(\int_{\Omega}\left[a(x)\left|u^{\prime}(x)\right|^{2}+|u(x)|^{2}\right] d x\right)^{\frac{1}{2}}, \quad \forall u \in H_{a}^{1}(\Omega) .
$$


Moreover, the Sobolev embedding theorem implies that $H_{a}^{1}(\Omega) \hookrightarrow C\left(\Omega_{0}\right)$, i.e. each element of $H_{a}^{1}(\Omega)$ is a continuous function in $\Omega \backslash\left\{x^{0}\right\}$.

We also introduce the closed subspace $H_{a, 0}^{1}(\Omega)$ of $H_{a}^{1}(\Omega)$ defined as

$$
H_{a, 0}^{1}(\Omega):=\left\{u \in H_{a}^{1}(\Omega): u(0)=0\right\} .
$$

We note that this subspace is correctly defined because the compactness of the embedding $H_{a, 0}^{1}(0, \varepsilon) \subset W_{0}^{1,2}((0, \varepsilon) ; 0) \hookrightarrow C([0, \varepsilon])$, for any $\varepsilon \in\left(0, x^{0}\right)$. So, if $u \in H_{a, 0}^{1}(\Omega)$, then $u(\cdot)$ is a continuous function at $x=0$, and, therefore, the condition $u(0)=0$ is consistent.

Let us show that, because of the degeneration of the weight function $a: \bar{\Omega} \rightarrow \mathbb{R}$ satisfying properties (i)-(ii), $H_{a, 0}^{1}(\Omega)$ is a Hilbert space with respect to the scalar product

$$
\langle u, v\rangle_{H_{a, 0}^{1}(\Omega)}=\int_{\Omega} a(x) u^{\prime}(x) v^{\prime}(x) d x+\int_{\Omega_{2}} u(x) v(x) d x, \quad \forall u, v \in H_{a, 0}^{1}(\Omega) .
$$

To do so, it is enough to establish some version of Poincaré inequality. We begin with the following observations.

Proposition 3.1. Let $a: \bar{\Omega} \rightarrow \mathbb{R}$ be a given function with properties (i)-(ii). Let $A_{i, a}$ and $\mu_{i, a}, i=1,2$, be the values given by relations (2.2), (2.3), and (2.4). Assume that

$$
0 \leq \max \left\{2 A_{1, a}, \mu_{1, a}\right\}, \max \left\{2 A_{2, a}, \mu_{2, a}\right\}<1 .
$$

Then each element of $H_{a}^{1}(\Omega)$ is an absolutely continuous function in $\bar{\Omega}$.

Proof. Let $u$ be an arbitrary element of $H_{a}^{1}(\Omega)$. As follows from Lemma 2.1, if assumption (3.3) holds true, then $1 / a(x) \in L^{1}(\Omega)$. Hence, in view of the representation

$$
u^{\prime}(x)=\frac{1}{\sqrt{a(x)}} \sqrt{a(x)} u_{x}(x), \quad \forall x \in \bar{\Omega}
$$

and the Cauchy-Bunjakovski inequality, we see that the function $u^{\prime}$ is summable over $\Omega$. Hence, $u(\cdot)$ is absolutely continuous in $\bar{\Omega}$.

Proposition 3.2. Let $a: \bar{\Omega} \rightarrow \mathbb{R}$ be a given function with properties (i)-(ii). Let $A_{i, a}$ and $\mu_{i, a}, i=1,2$, be the values given by relations (2.2), (2.3), and (2.4). Assume that the following conditions

$$
\max \left\{2 A_{1, a}, \mu_{1, a}\right\}<2 \text { and } \max \left\{2 A_{2, a}, \mu_{2, a}\right\}<2
$$

hold true. Then

$$
\begin{gathered}
\|u\|_{L^{2}\left(\Omega_{1}\right)} \leq \frac{1}{\left.\sqrt{a(0)\left[2-\max \left\{\mu_{1, a}, 2 A_{1, a}\right\}\right.}\right]}\|u\|_{H_{a}^{1}(\Omega)}, \\
\|u-u(1)\|_{L^{2}\left(\Omega_{2}\right)} \leq \frac{1}{\sqrt{a(1)\left[2-\max \left\{\mu_{2, a}, 2 A_{2, a}\right\}\right]}}\|u\|_{H_{a}^{1}(\Omega)} .
\end{gathered}
$$


Proof. Let $u$ be an arbitrary element of $H_{a, 0}^{1}(\Omega)$. Then, using direct arguments, for any $x \in\left[0, x^{0}\right)$, we have

$$
\begin{aligned}
|u(x)|=\left|\int_{0}^{x} u^{\prime}(s) d s\right| & =\left|\int_{0}^{x} \sqrt{a(s)} u^{\prime}(s) \frac{1}{\sqrt{a(s)}} d s\right| \\
& \leq\|u\|_{H_{a}^{1}(\Omega)}\left(\int_{0}^{x} \frac{d s}{a(s)}\right)^{\frac{1}{2}} .
\end{aligned}
$$

From this and estimate (2.7), by Fubini's theorem, we obtain

$$
\begin{aligned}
\|u\|_{L^{2}\left(\Omega_{1}\right)}^{2} & \leq\|u\|_{H_{a}^{1}(\Omega)}^{2} \int_{0}^{x^{0}} \int_{0}^{x} \frac{d s}{a(s)} d x \\
& =\|u\|_{H_{a}^{1}(\Omega)}^{2} \int_{0}^{x^{0}} \int_{s}^{x^{0}} d x \frac{d s}{a(s)} \\
& =\|u\|_{H_{a}^{1}(\Omega)}^{2} \int_{0}^{x^{0}} \frac{x^{0}-s}{a(s)} d s \\
& \text { by }{ }^{(2.7)}\|u\|_{H_{a}^{1}(\Omega)}^{2} \frac{1}{a(0)} \int_{0}^{x^{0}}\left(x^{0}-s\right)^{1-\max \left\{\mu_{1, a}, 2 A_{1, a}\right\}} d s \\
& =\frac{\|u\|_{H_{a}^{1}(\Omega)}^{2}\left(x^{0}\right)^{2-\max \left\{\mu_{1, a}, 2 A_{1, a}\right\}}}{a(0)\left[2-\max \left\{\mu_{1, a}, 2 A_{1, a}\right\}\right]} \\
& \leq \frac{\|u\|_{H_{a}^{1}(\Omega)}^{2}}{a(0)\left[2-\max \left\{\mu_{1, a}, 2 A_{1, a}\right\}\right]} .
\end{aligned}
$$

Arguing in a similar manner, for any $x \in\left(x^{0}, 1\right]$, we have

$$
|u(x)-u(1)|=\left|\int_{x}^{1} u^{\prime}(s) d s\right| \leq\|u\|_{H_{a}^{1}(\Omega)}\left(\int_{x}^{1} \frac{d s}{a(s)}\right)^{\frac{1}{2}} .
$$

Then, estimate (2.7) and Fubini's theorem yield the following bound

$$
\begin{aligned}
& \|u(\cdot)-u(1)\|_{L^{2}\left(\Omega_{2}\right)}^{2} \leq\|u\|_{H_{a}^{1}(\Omega)}^{2} \int_{x^{0}}^{1} \int_{x}^{1} \frac{d s}{a(s)} d x \\
& =\|u\|_{H_{a}^{1}(\Omega)}^{2} \int_{x^{0}}^{1} \int_{x^{0}}^{s} d x \frac{d s}{a(s)} \\
& =\|u\|_{H_{a}^{1}(\Omega)}^{2} \int_{x^{0}}^{1} \frac{s-x^{0}}{a(s)} d s \\
& \text { by } \stackrel{(2.8)}{\leq}\|u\|_{H_{a}^{1}(\Omega)}^{2} \frac{1}{a(1)} \int_{x^{0}}^{1}\left(s-x^{0}\right)^{1-\max \left\{\mu_{2, a}, 2 A_{2, a}\right\}} d s \\
& \leq \frac{\|u\|_{H_{a}^{1}(\Omega)}^{2}}{a(1)\left[2-\max \left\{\mu_{2, a}, 2 A_{2, a}\right\}\right]} .
\end{aligned}
$$


Proposition 3.3. Let $a: \bar{\Omega} \rightarrow \mathbb{R}$ be a given function with properties (i)-(ii). Let $A_{i, a}$ and $\mu_{i, a}, i=1,2$, be the values given by relations (2.2), (2.3), and (2.4), and satisfying restrictions (3.4). Then

$$
\|u\|_{L^{2}\left(\Omega_{1}\right)}^{2}+\|u-u(1)\|_{L^{2}\left(\Omega_{2}\right)}^{2} \leq\left[\frac{4}{a(0)}+\frac{4}{a(1)}\right]\|u\|_{H_{a}^{1}(\Omega)}^{2} .
$$

Proof. We adapt a reasoning here that can be used to prove Hardy's inequality. With that in mind, we observe that, for all $x \in\left[0, x^{0}\right]$, the following transformation is valid

$$
\begin{aligned}
\int_{0}^{x}\left(x^{0}-s\right) u^{\prime}(s) u(s) d s & =\frac{1}{2} \int_{0}^{x}\left(x^{0}-s\right) \frac{d}{d s} u^{2}(s) d x \\
& =\frac{1}{2}\left(x^{0}-x\right) u^{2}(x)+\frac{1}{2} \int_{0}^{x} u^{2}(s) d x .
\end{aligned}
$$

Hence,

$$
\begin{aligned}
0 & \leq \int_{0}^{x}\left[\left(x^{0}-s\right) u^{\prime}(s)-\frac{1}{2} u(s)\right]^{2} d s \\
& =\int_{0}^{x}\left[\left(x^{0}-s\right)^{2}\left[u^{\prime}(s)\right]^{2}+\frac{1}{4} u^{2}(s)-\left(x^{0}-s\right) u^{\prime}(s) u(s)\right] d s \\
& \text { by } \stackrel{(3.10)}{=} \int_{0}^{x}\left[\left(x^{0}-s\right)^{2}\left[u^{\prime}(s)\right]^{2}-\frac{1}{4} u^{2}(s)\right] d s-\frac{1}{2}\left(x^{0}-x\right) u^{2}(x) .
\end{aligned}
$$

From this, we deduce that

$$
\begin{aligned}
\int_{0}^{x} u^{2}(s) d s & \leq 4 \int_{0}^{x}\left(x^{0}-s\right)^{2}\left[u^{\prime}(s)\right]^{2} d s \\
& \text { by } \stackrel{(3.4)}{\leq} 4 \int_{0}^{x}\left(x^{0}-s\right)^{\max \left\{2 A_{1, a}, \mu_{1, a}\right\}}\left[u^{\prime}(s)\right]^{2} d s \\
& \text { by } \stackrel{(2.7)}{\leq} \frac{4}{a(0)} \int_{0}^{x} a(s)\left[u^{\prime}(s)\right]^{2} d s .
\end{aligned}
$$

Taking the limit as $x \uparrow x^{0}$ in the last relation, we arrive at the estimate

$$
\|u\|_{L^{2}\left(\Omega_{1}\right)}^{2} \leq \frac{4}{a(0)}\|u\|_{H_{a}^{1}(\Omega)}^{2} .
$$

By analogy with the previous case, we make use of the following transformation which is valid for each $x \in\left[x^{0}, 1\right]$.

$$
\begin{array}{r}
\int_{x}^{1}\left(s-x^{0}\right)(u(s)-u(1)) u^{\prime}(s) d s=\frac{1}{2} \int_{x}^{1}\left(s-x^{0}\right) \frac{d}{d s}(u(s)-u(1))^{2} d s \\
=\frac{1}{2}\left[-\left(x-x^{0}\right)(u(x)-u(1))^{2}-\int_{x}^{1}(u(s)-u(1))^{2} d s\right] .
\end{array}
$$


Then

$$
\begin{aligned}
& 0 \leq \int_{x}^{1}\left[\left(s-x^{0}\right) u^{\prime}(s)+\frac{1}{2}(u(s)-u(1))\right]^{2} d s \\
& =\int_{x}^{1}\left[\left(s-x^{0}\right)^{2}\left[u^{\prime}(s)\right]^{2}+\frac{1}{4}(u(s)-u(1))^{2}\right] d s \\
& +\int_{x}^{1}\left(s-x^{0}\right)(u(s)-u(1)) u^{\prime}(s) d s \\
& \text { by } \stackrel{(3.12)}{=} \int_{x}^{1}\left[\left(s-x^{0}\right)^{2}\left[u^{\prime}(s)\right]^{2}-\frac{1}{4}(u(s)-u(1))^{2}\right] d s \\
& -\frac{1}{2}\left(x-x^{0}\right)(u(x)-u(1))^{2} \text {. }
\end{aligned}
$$

Since $\left(x-x^{0}\right)(u(x)-u(1))^{2} \geq 0$ for all $x \in\left[x^{0}, 1\right]$, it follows that

$$
\begin{aligned}
& \int_{x}^{1}(u(s)-u(1))^{2} d s \leq 4 \int_{x}^{1}\left(s-x^{0}\right)^{2}\left[u^{\prime}(s)\right]^{2} d s
\end{aligned}
$$

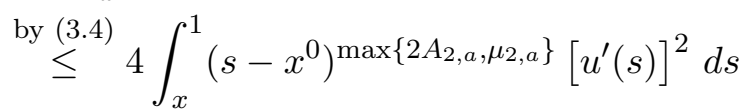

$$
\begin{aligned}
& \stackrel{\text { by }}{\leq} \frac{4}{a(1)} \int_{x}^{1} a(s)\left[u^{\prime}(s)\right]^{2} d s \text {. }
\end{aligned}
$$

As a result, passing to the limit in the last relation as $x \downarrow x^{0}$, we arrive at the following inequality

$$
\|u-u(1)\|_{L^{2}\left(\Omega_{2}\right)}^{2} \leq \frac{4}{a(1)}\|u\|_{H_{a}^{1}(\Omega)}^{2} .
$$

Thus, the announced estimate (3.9) is a direct consequence of (3.11) and (3.13).

Before proceeding further, we notice that if $u$ is an arbitrary element of the standard Sobolev space $W^{1,2}(d, 1)$ with $d \in\left(x^{0}, 1\right)$, then $u(\cdot)$ is an absolutely continuous function on $[d, 1]$. Moreover, by Sobolev embedding theorem, the injection $W^{1,2}(d, 1) \hookrightarrow C^{0,1}([d, 1])$ is continuous and there exists a constant $C_{S o b}>0$ such that

$$
\max _{x \in[d, 1]}|u(x)| \leq C_{S o b}\|u\|_{W^{1,2}(d, 1)}, \quad \forall u \in W^{1,2}(d, 1) .
$$

Taking this fact into account and fixing an arbitrary element $u \in H_{a, 0}^{1}(\Omega)$ and 
$d \in\left(x^{0}, 1\right)$, we note that $u \in W^{1,2}(d, 1)$ and

$$
\begin{aligned}
\|u\|_{W^{1,2}(d, 1)}^{2} & =\int_{d}^{1}\left[u^{2}(s)+\left[u^{\prime}(s)\right]^{2}\right] d s \\
& =\int_{d}^{1}\left[u^{2}(s)+\frac{1}{a^{2}(s)}\left[a(s) u^{\prime}(s)\right]^{2}\right] d s \\
& \leq\left(1+\max _{x \in[d, 1]} \frac{1}{a^{2}(x)}\right)\|u\|_{H_{a, 0}^{1}(\Omega)}^{2} .
\end{aligned}
$$

Utilizing this estimate together with (3.14), we get

$$
\begin{aligned}
|u(1)| \leq\|u\|_{C([d, 1])} & \stackrel{\text { by }}{(3.14)} C_{S o b}\|u\|_{W^{1,2}(d, 1)} \\
\leq & C_{S o b} \sqrt{1+\max _{x \in[d, 1]} \frac{1}{a^{2}(x)}}\|u\|_{H_{a, 0}^{1}(\Omega)} .
\end{aligned}
$$

Since $d$ is an arbitrary point of the interval $\left(x^{0}, 1\right)$, we can pass to the limit in (3.15) as $d \uparrow 1$. As a result, we obtain

$$
|u(1)| \leq C_{S o b} \sqrt{1+\frac{1}{a^{2}(1)}}\|u\|_{H_{a, 0}^{1}(\Omega)}, \quad \forall u \in H_{a, 0}^{1}(\Omega) .
$$

We are now in a position to prove the main result of this section. Namely, we establish some variant of Poincaré's (or Friedrich's) inequality for weighted Sobolev space $H_{a, 0}^{1}(\Omega)$ and derive the conditions when this inequality is consistent.

Theorem 3.1. Let $a: \bar{\Omega} \rightarrow \mathbb{R}$ be a given weight function with properties (i)-(ii). Let $A_{i, a}$ and $\mu_{i, a}, i=1,2$, be the values given by relations (2.2), (2.3), and (2.4), and satisfying conditions (3.4). Then

$$
\|u\|_{L^{2}(\Omega)} \leq\left[C_{1}+C_{2}\right]\|u\|_{H_{a}^{1}(\Omega)}, \quad \forall u \in H_{a, 0}^{1}(\Omega),
$$

where

$$
\begin{aligned}
& C_{1}=\frac{1}{\sqrt{a(0)}} \min \left\{\frac{1}{\left.\sqrt{\left[2-\max \left\{\mu_{1, a}, 2 A_{1, a}\right\}\right.}\right]}, 2\right\}, \\
& C_{2}=\frac{1}{\sqrt{a(1)}}\left[\min \left\{\frac{1}{\sqrt{\left[2-\max \left\{\mu_{2, a}, 2 A_{2, a}\right\}\right]}}, 2\right\}+C_{S o b} \sqrt{1+a^{2}(1)}\right] .
\end{aligned}
$$

Proof. By Propositions 3.2 and 3.3, the following estimate

$$
\|u-u(1)\|_{L^{2}\left(\Omega_{2}\right)} \leq \frac{1}{\sqrt{a(1)}} \min \left\{\frac{1}{\sqrt{\left[2-\max \left\{\mu_{2, a}, 2 A_{2, a}\right\}\right]}}, 2\right\}\|u\|_{H_{a}^{1}(\Omega)}
$$


holds true for any $u \in H_{a, 0}^{1}(\Omega)$. Since

$$
\|u\|_{L^{2}\left(\Omega_{2}\right)} \leq\|u-u(1)\|_{L^{2}\left(\Omega_{2}\right)}+\sqrt{1-x^{0}}|u(1)|,
$$

it follows from (3.16) and (3.18) that

$$
\begin{aligned}
\|u\|_{L^{2}\left(\Omega_{2}\right)} \leq \frac{\|u\|_{H_{a}^{1}(\Omega)}}{\sqrt{a(1)}}\left[\min \left\{\frac{1}{\sqrt{\left[2-\max \left\{\mu_{2, a}, 2 A_{2, a}\right\}\right]}}, 2\right\}\right. \\
\left.+C_{S o b} \sqrt{1+a^{2}(1)}\right]
\end{aligned}
$$

To derive the announced Friedrichs inequality (3.17), it remains to utilize relations (3.5)-(3.6) and (3.9) and combine them with (3.19).

As an obvious consequence of this theorem, we can give the following conclusion.

Corollary 3.1. Under the assumptions of Theorem 3.1, $H_{a, 0}^{1}(\Omega)$ is a Banach space with respect to the norm

$$
\|u\|_{H_{a, 0}^{1}(\Omega)}=\left(\int_{\Omega}\left[a(x)\left|u^{\prime}(x)\right|^{2}\right] d x\right)^{\frac{1}{2}} .
$$

However, as it will be shown in the next section, the expression (3.20) can lose the norm properties in $H_{a, 0}^{1}(\Omega)$ if assumptions of Theorem 3.1 are not valid..

The next result is crucial for our further consideration and it reveals an alternative way to the substantiation of the Friedrich's inequality (3.17).

Theorem 3.2. Let $a: \bar{\Omega} \rightarrow \mathbb{R}$ be a function such that, in addition to the properties (i)-(ii), it satisfies the following ones

(iii) there exists subintervals $\left(x_{1}^{*}, x^{0}\right) \subset \Omega_{1}$ and $\left(x^{0}, x_{2}^{*}\right) \subset \Omega_{2}$ such that $a(\cdot)$ is monotonically decreasing on $\left(x_{1}^{*}, x^{0}\right)$ and it is a monotonically increasing function on $\left(x^{0}, x_{2}^{*}\right)$;

(iv) $(\sqrt{a})_{x} \neq$ const in $\Omega$ and $(\sqrt{a})_{x}^{-1} \in L^{\infty}(\Omega)$.

Then inequalities (3.4) holds true, where $A_{i, a}$ and $\mu_{i, a}, i=1,2$, are the values given by relations (2.2), (2.3), and (2.4).

Proof. Let $a: \bar{\Omega} \rightarrow \mathbb{R}$ be a given function with properties (i)-(iv). Setting

$$
k:=\left\|(\sqrt{a})_{x}^{-1}\right\|_{L^{\infty}(\Omega)}^{2} \quad \text { and } \quad \widehat{a}(x):=k a(x) \text { for all } x \in \Omega,
$$

we see that the function $\widehat{a}: \bar{\Omega} \rightarrow \mathbb{R}$ possesses all properties (i)-(iv) and the direct calculations show that

$$
\mu_{i, a}=\mu_{i, \widehat{a}} \quad \text { and } \quad A_{i, a}=A_{i, \widehat{a}}, \quad i=1,2 .
$$


Moreover, in this case, we have

$$
\left\|(\sqrt{\widehat{a}})_{x}^{-1}\right\|_{L^{\infty}(\Omega)}=\frac{1}{\sqrt{k}}\left\|(\sqrt{a})_{x}^{-1}\right\|_{L^{\infty}(\Omega)}=1 .
$$

Hence, without loss of generality, we can suppose that the function $a: \bar{\Omega} \rightarrow \mathbb{R}$ is such that

$$
\left\|(\sqrt{a})_{x}^{-1}\right\|_{L^{\infty}(\Omega)} \leq 1
$$

As a consequence of this condition, we have

$$
\sup _{x \in\left[0, x^{0}\right)} \frac{2 \sqrt{a(x)}}{\left|a^{\prime}(x)\right|} \leq 1 \quad \text { and } \sup _{x \in\left(x^{0}, 1\right]} \frac{2 \sqrt{a(x)}}{\left|a^{\prime}(x)\right|} \leq 1 .
$$

So, we can suppose that

$$
2 \sqrt{a(x)} \leq\left|a^{\prime}(x)\right|, \quad \forall x \in\left[x_{1}^{*}, x_{2}^{*}\right] .
$$

Since $a(\cdot)$ is a monotonically decreasing function on $\left(x_{1}^{*}, x^{0}\right)$ and $a(\cdot)$ is a monotonically increasing function on $\left(x^{0}, x_{2}^{*}\right)$, it follows from (3.23) that

$$
a^{\prime}(x) \leq-2 \sqrt{a(x)}, \quad \forall x \in\left[x_{1}^{*}, x^{0}\right) \quad \text { and } \quad a^{\prime}(x) \geq 2 \sqrt{a(x)}, \quad \forall x \in\left(x^{0}, x_{2}^{*}\right] .
$$

Then, after integration, we obtain

$$
\begin{gathered}
\int_{x}^{x^{0}} \frac{a^{\prime}(s)}{\sqrt{a(s)}} d s \leq-2\left(x^{0}-x\right), \quad \forall x \in\left[x_{1}^{*}, x^{0}\right), \\
\int_{x^{0}}^{x} \frac{a^{\prime}(s)}{\sqrt{a(s)}} d s \geq 2\left(x-x^{0}\right), \quad \forall x \in\left(x^{0}, x_{2}^{*}\right] .
\end{gathered}
$$

Taking into account that $a\left(x^{0}\right)=0$, we deduce from (3.24)-(3.25) that

$$
\sqrt{a(x)} \geq x^{0}-x, \quad \forall x \in\left[x_{1}^{*}, x^{0}\right) \quad \text { and } \quad \sqrt{a(x)} \geq x-x^{0}, \quad \forall x \in\left(x^{0}, x_{2}^{*}\right],
$$

and, as a consequence, we have

$$
a(x) \geq\left(x-x^{0}\right)^{2}, \quad \forall x \in\left[x_{1}^{*}, x_{2}^{*}\right] .
$$

Utilizing the monotonicity property of $a(\cdot)$ around the point $x^{0}$, we deduce from (3.26) that there exists a positive value $\gamma \in(0,2)$ such that

$$
a(x)=\mathcal{O}\left(\left|x-x^{0}\right|^{2-\gamma}\right) \quad \text { in }\left[x_{1}^{*}, x_{2}^{*}\right],
$$

that is, $a(x) \sim\left|x-x^{0}\right|^{2-\gamma}$ near the degeneration point $x^{0}$. Therefore, in view of representation $(2.4)$, we have

$$
\begin{aligned}
& \mu_{1, a}:=\sup _{x \in \Omega_{1}} \frac{\left|x-x^{0}\right|\left|a^{\prime}(x)\right|}{a(x)}=\sup _{x \in\left[x_{1}^{*}, x^{0}\right)} \frac{\left|x-x^{0}\right|\left|a^{\prime}(x)\right|}{a(x)}=2-\gamma \leq 2, \\
& \mu_{2, a}:=\sup _{x \in \Omega_{2}} \frac{\left|x-x^{0}\right|\left|a^{\prime}(x)\right|}{a(x)}=\sup _{x \in\left(x^{0}, x_{2}^{*}\right]} \frac{\left|x-x^{0}\right|\left|a^{\prime}(x)\right|}{a(x)}=2-\gamma \leq 2 .
\end{aligned}
$$


Since for the functions $a: \bar{\Omega} \rightarrow \mathbb{R}$ with property (3.27), we can set $G_{i}(x)=x$, $i=1,2$, it follows from (2.2)-(2.3) and (3.28)-(3.29) that

$$
2 A_{i, a}=\mu_{i, a} \leq 2 .
$$

It remains to notice that initial assumption $(\sqrt{a})_{x} \neq C_{*}=$ const leads to the relation

$$
a(x) \neq C_{*}^{2}\left(x-x^{0}\right)^{2} \quad \text { in } \Omega .
$$

As a result, we have: $\mu_{i, a} \neq 2$ for $i=1,2$. Combining this fact with (3.30), we arrive at the inequalities (3.4).

Example 3.1. Let the degenerate weight $a: \bar{\Omega} \rightarrow \mathbb{R}_{+}$be defined by the rule (2.5). Then

$$
(\sqrt{a(x)})_{x}= \begin{cases}-p_{1}\left(\frac{1}{2}-x\right)^{p_{1}-1}, & \text { if } x \in\left[0, \frac{1}{2}\right] \\ p_{2}\left(x-\frac{1}{2}\right)^{p_{2}-1}, & \text { if } x \in\left(\frac{1}{2}, 1\right] .\end{cases}
$$

Therefore, conditions (iii)-(iv) are satisfied with $0<p_{1}, p_{2}<1$. Hence,

$$
2 A_{i, a} \leq \mu_{i, a}<2, \quad i=1,2,
$$

and this statement can be approved by the direct calculations. At the same time, if the weight function $a:[0,1] \rightarrow \mathbb{R}_{+}$is defined as in $(2.6)$, then $(\sqrt{a})_{x}^{-1} \notin L^{\infty}(\Omega)$ for any $p>0$. In this case, as it is indicated in Example 2.1, we have $\mu_{i, a}=+\infty$ for $i=1,2$.

\section{Other Weighted Sobolev Spaces Associated with Degenerate Weight Functions}

We begin this section with the following assumptions on function $a: \bar{\Omega} \rightarrow \mathbb{R}_{+}$.

Definition 4.1. We say that the function $a: \bar{\Omega} \rightarrow \mathbb{R}_{+}$has a very strong degeneration at point $x=x^{0} \in \Omega$ if

(j) $a\left(x^{0}\right)=0$ and $a(x)>0$ for all $x \in \bar{\Omega} \backslash\left\{x^{0}\right\}$;

(jj) $a \in C(\bar{\Omega}) \cap C_{l o c}^{1}\left(\Omega_{0}\right)$;

(jjj) there exists subintervals $\left(x_{1}^{*}, x^{0}\right) \subset \Omega_{1}$ and $\left(x^{0}, x_{2}^{*}\right) \subset \Omega_{2}$ such that $a(\cdot)$ is monotonically decreasing on $\left(x_{1}^{*}, x^{0}\right)$ and it is a monotonically increasing function on $\left(x^{0}, x_{2}^{*}\right)$;

(jv) $(\sqrt{a})_{x} \in L^{\infty}(\Omega)$. 
In what follows, we associate with a such function $a(\cdot)$ the following space

$$
V_{a, 0}(\Omega)=\left\{y \in L^{2}(\Omega): y(0)=0, \sqrt{a} y \in W_{0}^{1,2}(\Omega ; 0)\right\} .
$$

We note that this space is correctly defined because the compactness of the embedding $W_{0}^{1,2}(\Omega ; 0) \hookrightarrow C(\bar{\Omega})$. Indeed, if $y \in V_{a, 0}(\Omega)$ then $u=\sqrt{a} y \in W_{0}^{1,2}(\Omega ; 0)$, and, therefore, $u(\cdot)$ is an absolutely continuous function on $\bar{\Omega}=[0,1]$. Thus, in view of $(\mathrm{j})$-property of $a(\cdot), y(x)=\frac{u(x)}{\sqrt{a(x)}}$ is a continuous function at $x=0$, so the condition $y(0)=0$ is consistent.

Proposition 4.1. $V_{a, 0}(\Omega)$ is a Banach space with respect to the norm

$$
\|y\|_{V_{a, 0}(\Omega)}=\left(\|y\|_{L^{2}(\Omega)}^{2}+\left\|(\sqrt{a} y)_{x}\right\|_{L^{2}(\Omega)}^{2}\right)^{1 / 2} .
$$

Proof. Let $\left\{y_{k}\right\}_{k \in \mathbb{N}}$ be a Cauchy sequence in $V_{a, 0}(\Omega)$. Then $\left\{\left(\sqrt{a} y_{k}\right)_{x}\right\}_{k \in \mathbb{N}}$ and $\left\{y_{k}\right\}_{k \in \mathbb{N}}$ are Cauchy sequences in $L^{2}(\Omega)$, which is the Banach space. Consequently, there exist elements $y \in L^{2}(\Omega)$ and $w \in L^{2}(\Omega)$ such that

$$
\begin{aligned}
y_{k} & \rightarrow y \text { strongly in } L^{2}(\Omega), \\
\left(\sqrt{a} y_{k}\right)_{x} & \rightarrow w \text { strongly in } L^{2}(\Omega) .
\end{aligned}
$$

Let us show that $w=\nabla(\sqrt{a} y)$. With that in mind, we set

$$
v_{k}=\sqrt{a} y_{k}, \quad \forall k \in \mathbb{N} .
$$

Since $v_{k} \in W_{0}^{1,2}(\Omega ; 0)$, it follows from the definition of the norm $\|v\|_{W_{0}^{1,2}(\Omega ; 0)}:=$ $\left(\int_{\Omega}|\nabla v|^{2} d x\right)^{1 / 2}$ in $W_{0}^{1,2}(\Omega ; 0)$ that $\left\{v_{k}\right\}_{k \in \mathbb{N}}$ is a Cauchy sequence in $W_{0}^{1,2}(\Omega ; 0)$. Therefore, in view of the compact embedding $W_{0}^{1,2}(\Omega ; 0) \hookrightarrow L^{2}(\Omega)$, we can suppose that, for some $v \in W_{0}^{1,2}(\Omega ; 0)$,

$$
\begin{gathered}
v_{k} \rightarrow v \text { strongly in } W_{0}^{1,2}(\Omega ; 0) \text { as } k \rightarrow \infty, \text { and } \\
v_{k} \rightarrow v \text { strongly in } L^{2}(\Omega) .
\end{gathered}
$$

Since $\sqrt{a} y \in L^{2}(\Omega)$ by property (i), it follows that

$$
\begin{aligned}
\|\sqrt{a} y-v\|_{L^{2}(\Omega)} & \leq\left\|\sqrt{a} y-\sqrt{a} y_{k}\right\|_{L^{2}(\Omega)}+\left\|v_{k}-v\right\|_{L^{2}(\Omega)} \\
& \leq \sqrt{\|a\|_{C(\bar{\Omega})}}\left\|y_{k}-y\right\|_{L^{2}(\Omega)}+C\left\|v_{k}-v\right\|_{W_{0}^{1,2}(\Omega ; 0)} \rightarrow 0 \text { as } k \rightarrow \infty
\end{aligned}
$$

Thus,

$$
v=\sqrt{a} y \text { in } \bar{\Omega}
$$

and this implies the equality

$$
v_{x}=(\sqrt{a} y)_{x} \stackrel{\text { by }}{\stackrel{(4.3)}{=}} w .
$$

The fact that the element $y(\cdot)$ has zero trace at $x=0$ is a direct consequence of (4.2) and definition of the space $V_{a, 0}(\Omega)(4.1)$. 
As immediately follows from (4.1) and Proposition 4.1, $V_{a, 0}(\Omega)$ is a Hilbert space with respect to the inner product

$$
\left(y_{1}, y_{2}\right)_{V_{a}(\Omega)}=\left(y_{1}, y_{2}\right)_{L^{2}(\Omega)}+\left(\left(\sqrt{a} y_{1}\right)_{x},\left(\sqrt{a} y_{2}\right)_{x}\right)_{L^{2}(\Omega)}, \quad \forall y_{1}, y_{2} \in V_{a, 0}(\Omega) .
$$

It is worth to emphasize that, in general, conditions $(\mathrm{j})-(\mathrm{jv})$, that we postulate for the function $a: \bar{\Omega} \rightarrow \mathbb{R}_{+}$, do not guarantee fulfillment of the inclusion $V_{a, 0}(\Omega) \subset C(\bar{\Omega})$ (see Proposition 3.1 for comparison). Indeed, in spite of the fact that the inclusion $y \in V_{a, 0}(\Omega)$ implies the property $\varphi:=\sqrt{a} y \in C^{0,1 / 2}(\bar{\Omega})$, we see that the function $y=\frac{1}{\sqrt{a}} \varphi$ is absolutely continuous in $\Omega \backslash\left\{x^{0}\right\}$ and, therefore, $y(\cdot)$ can have a gap at $x=x^{0}$. So, if $y \in V_{a, 0}(\Omega)$, then $y \in C(\bar{\Omega})$ provided

$$
\lim _{x \nearrow x^{0}} y(x)=\lim _{x \searrow x^{0}} y(x) \text { and } \sup _{x \in \Omega}|y(x)|<+\infty .
$$

Our next result concerns the comparison of the weighted space $V_{a, 0}(\Omega)$ and the space that $H_{a}^{1}(\Omega)$ that has been considered in the previous section. It is clear that, in general, the spaces $H_{a}^{1}(\Omega)$ and $V_{a, 0}(\Omega)$ differ from each other. However, due to $(\mathrm{j})-(\mathrm{jv})$ properties of the weight function $a(\cdot)$, we can establish the following result.

Theorem 4.1. Let $a: \bar{\Omega} \rightarrow \mathbb{R}$ be a weight function with properties $(j)-(j v)$. Then the norms $\|\cdot\|_{V_{a}(\Omega)}$ and $\|\cdot\|_{H_{a}^{1}(\Omega)}$ are equivalent on $H_{a}^{1}(\Omega)$.

Proof. Let $y$ be an element of $H_{a}^{1}(\Omega)$. Then taking into account the relation

$$
(\sqrt{a} y)_{x}=(\sqrt{a})_{x} y+\sqrt{a} y_{x} \text { for a.a. } x \in \Omega,
$$

we see that

$$
\left.\left.\|y\|_{V_{a, 0}(\Omega)}^{2} \leq\|y\|_{L^{2}(\Omega)}^{2}+2 \|(\sqrt{a})_{x} y\right)\left\|_{L^{2}(\Omega)}^{2}+2\right\| \sqrt{a} y_{x}\right) \|_{L^{2}(\Omega)}^{2} .
$$

Using the fact that $(\sqrt{a})_{x} \in L^{\infty}(\Omega)$, we arrive the estimate

$$
\|y\|_{V_{a, 0}(\Omega)}^{2} \leq \max \left\{2,1+2\left\|(\sqrt{a})_{x}\right\|_{L^{\infty}(\Omega)}\right\}\|y\|_{H_{a}^{1}(\Omega)}^{2} .
$$

On the other hand, representation (4.5) implies that

$$
\begin{aligned}
\|y\|_{H_{a}^{1}(\Omega)}^{2} & \left.\left.\leq\|y\|_{L^{2}(\Omega)}^{2}+2 \|(\sqrt{a})_{x} y\right)\left\|_{L^{2}(\Omega)}^{2}+2\right\|(\sqrt{a} y)_{x}\right) \|_{L^{2}(\Omega)}^{2} \\
& \leq \max \left\{2,1+2\left\|(\sqrt{a})_{x}\right\|_{L^{\infty}(\Omega)}\right\}\|y\|_{V_{a, 0}(\Omega)}^{2} .
\end{aligned}
$$

Hence,

$$
C_{H}^{-1}\|y\|_{H_{a}^{1}(\Omega)} \leq\|y\|_{V_{a, 0}(\Omega)} \leq C_{H}\|y\|_{H_{a}^{1}(\Omega)},
$$

with

$$
C_{H}=\left(\max \left\{2,1+2\left\|(\sqrt{a})_{x}\right\|_{L^{\infty}(\Omega)}\right\}\right)^{\frac{1}{2}},
$$

and announced equivalence of the norms follows. 
The next observations are crucial for our further analysis.

Lemma 4.1. Let $a: \bar{\Omega} \rightarrow \mathbb{R}$ be a function with properties $(j)-(j v)$, and let $W_{a}(\Omega)$ be a vector space defined as follows

$$
W_{a}(\Omega):=\left\{y: \Omega \rightarrow \mathbb{R} \text { is measurable and } \sqrt{a} y \in W_{0}^{1,2}(\Omega ; 0)\right\} .
$$

Then

$$
\|y\|_{W_{a}(\Omega)}:=\left(\int_{\Omega}\left[(\sqrt{a} y)_{x}\right]^{2} d x\right)^{1 / 2}
$$

is the norm on $W_{a}(\Omega)$.

Proof. As it follows from (4.8), the function $\|\cdot\|_{W_{a}(\Omega)}$ is subadditive and absolutely scalable, that is, $\|\cdot\|_{W_{a}(\Omega)}$ is a seminorm on $W_{a}(\Omega)$. Let us show that this function is point-separating, i.e., the condition $\|y\|_{W_{a}(\Omega)}=0$ guarantees the following equalities

$$
y(x)=0 \quad \text { and } \quad y_{x}(x)=0 \quad \text { a.e. in } \Omega .
$$

Indeed, if for some $y \in W_{a}(\Omega)$ we have $\|y\|_{W_{a}(\Omega)}=0$, then the Friedrich's inequality

$$
\|\sqrt{a} y\|_{L^{2}(\Omega)}^{2} \leq C_{F} \int_{\Omega}\left|(\sqrt{a} y)_{x}\right|^{2} d x
$$

ensures the validity of the relation

$$
\|\sqrt{a} y\|_{L^{2}(\Omega)}=0 .
$$

Hence, in view of the properties $(\mathrm{j})-(\mathrm{jj})$ of function $a(\cdot)$, we deduce from $(4.10)$ that

$$
y(x)=0 \quad \text { a.e. in } \Omega .
$$

Taking this fact into account, we obtain

$$
\begin{aligned}
0 & =\|\sqrt{a} y\|_{L^{2}(\Omega)}^{2}=\int_{\Omega}\left[(\sqrt{a} y)_{x}\right]^{2} d x \\
& =\int_{\Omega}\left[(\sqrt{a})_{x} y+\sqrt{a} y_{x}\right]^{2} d x=I_{1}+I_{2}+\int_{\Omega} a y_{x}^{2} d x
\end{aligned}
$$

where

$$
\begin{aligned}
& 0 \leq\left|I_{1}\right|=\left|\int_{\Omega}(\sqrt{a})_{x}^{2} y^{2} d x\right| \leq\left\|(\sqrt{a})_{x}\right\|_{L^{\infty}(\Omega)}^{2}\|y\|_{L^{2}(\Omega)}^{2} \stackrel{\text { by }}{=} \stackrel{(4.11)}{=}^{\prime} 0, \\
& 0 \leq\left|I_{2}\right|=\left|2 \int_{\Omega} \sqrt{a}(\sqrt{a})_{x} y y_{x} d x\right| \leq 2\left\|(\sqrt{a})_{x}\right\|_{L^{\infty}(\Omega)}\left\|\sqrt{a} y_{x}\right\|_{L^{2}(\Omega)}\|y\|_{L^{2}(\Omega)} \\
& \text { by } \stackrel{(4.5)}{\leq} 2\left\|(\sqrt{a})_{x}\right\|_{L^{\infty}(\Omega)}\|y\|_{L^{2}(\Omega)}\left(\left\|(\sqrt{a} y)_{x}\right\|_{L^{2}(\Omega)}+\left\|(\sqrt{a})_{x}\right\|_{L^{\infty}(\Omega)}\|y\|_{L^{2}(\Omega)}\right) \\
& \quad \text { by } \stackrel{(4.11)}{=} 0 .
\end{aligned}
$$


Hence, in view of representation (4.12), we deduce

$$
\int_{\Omega} a y_{x}^{2} d x=0
$$

Since $a(\cdot)$ vanishes at a single point $x=x^{0}$, we finally have $y_{x}(x)=0$ a.e. in $\Omega$.

As for the Friedrich's inequality (4.9), we notice that in view of the following estimate

$$
\begin{aligned}
\int_{\Omega} u^{2} d x= & \left.(x-1) u^{2}(x)\right|_{0} ^{1}-2 \int_{0}^{1}(x-1) u u_{x} d x \\
& \text { by inequality }-c b \leq \frac{c^{2}}{2}+\frac{b^{2}}{2} \\
\leq & -u^{2}(0)+\frac{1}{2} \int_{\Omega} u^{2} d x+2 \int_{0}^{1}(x-1)^{2} u_{x}^{2} d x \\
\leq & u^{2}(0)+\frac{1}{2} \int_{\Omega} u^{2} d x+2 \int_{0}^{1} u_{x}^{2} d x,
\end{aligned}
$$

we see that

$$
\int_{\Omega} u^{2} d x \leq 4 \int_{0}^{1} u_{x}^{2} d x, \quad \forall u \in W_{0}^{1,2}(\Omega ; 0) .
$$

Hence, the constant $C_{F}$ in (4.9) is equal to 4 .

Lemma 4.2. Let $a: \bar{\Omega} \rightarrow \mathbb{R}$ be a function with properties $(j)-(j v)$. Then the injection $W_{0}^{1,2}(\Omega ; 0) \hookrightarrow W_{a}(\Omega)$ is continuous.

Proof. Let $u$ be an element of the standard Sobolev space $W_{0}^{1,2}(\Omega ; 0)$. Then

$$
\begin{aligned}
& \|u\|_{W_{a}(\Omega)}^{2}:=\int_{\Omega}\left[(\sqrt{a} u)_{x}\right]^{2} d x \leq 2 \int_{\Omega}\left[(\sqrt{a})_{x}^{2} u^{2}+a u_{x}^{2}\right] d x \\
& \quad \stackrel{\text { by (jv)-property }}{\leq} 2\left[\left\|(\sqrt{a})_{x}\right\|_{L^{\infty}(\Omega)}^{2}\|u\|_{L^{2}(\Omega)}^{2}+\|a\|_{C(\Omega)}\left\|u_{x}\right\|_{L^{2}(\Omega)}^{2}\right] \\
& \quad \stackrel{\leq}{\quad} 2\left(\left\|(\sqrt{a})_{x}\right\|_{L^{\infty}(\Omega)}^{2} C_{F}+\|a\|_{C(\Omega)}\right)\|u\|_{W_{0}^{1,2}(\Omega ; 0)}^{2} .
\end{aligned}
$$

The proof is complete.

Taking this result into account, we make use of the following Banach space

$$
\widehat{V}_{a, 0}(\Omega)=\text { closure }_{\|\cdot\|_{W_{a}(\Omega)}}\left\{\varphi \in C_{0}^{\infty}(\mathbb{R}): \varphi(0)=0\right\},
$$

which is a Hilbert space with respect to the inner product

$$
\left(y_{1}, y_{2}\right)_{\widehat{V}_{a, 0}(\Omega)}=\left(\left(\sqrt{a} y_{1}\right)_{x},\left(\sqrt{a} y_{2}\right)_{x}\right)_{L^{2}(\Omega)}, \quad \forall y_{1}, y_{2} \in \widehat{V}_{a, 0}(\Omega) .
$$

It is clear now that

$$
H_{a, 0}^{1}(\Omega) \stackrel{\text { by Theorem }}{=}{ }^{4.1} V_{a, 0}(\Omega)=\widehat{V}_{a, 0}(\Omega) \cap L^{2}(\Omega) .
$$

Utilizing this representation, Theorem 4.1, and the Friedrich's inequality (4.9) with $C_{F}=4$ (see (4.14)), we can give the following conclusion. 
Theorem 4.2. Let $a: \bar{\Omega} \rightarrow \mathbb{R}$ be a function with properties (j)-(jv). Then

$$
\|\sqrt{a} u\|_{L^{2}(\Omega)} \leq 2\left\|(\sqrt{a} u)_{x}\right\|_{L^{2}(\Omega)} \leq 2 \sqrt{2}\left(1+\left\|(\sqrt{a})_{x}\right\|_{L^{\infty}(\Omega)}\right)^{\frac{1}{2}}\|u\|_{H_{a}^{1}(\Omega)},
$$

for all elements $u \in H_{a, 0}^{1}(\Omega)$.

Proof. Let $y \in H_{a, 0}^{1}(\Omega)$ be an arbitrary element. Then $y \in V_{a, 0}^{1}(\Omega)$ by Theorem 4.1, and hence $\sqrt{a} y \in W_{0}^{1,2}(\Omega ; 0)$. As a result, utilizing inequalities (4.9) and (4.6), we obtain

$$
\|\sqrt{a} y\|_{L^{2}(\Omega)} \leq 2\left\|(\sqrt{a} y)_{x}\right\|_{L^{2}(\Omega)} \leq 2\|y\|_{V_{a}^{1}(\Omega)} \leq 2 C_{H}\|y\|_{H_{a}^{1}(\Omega)} .
$$

It remains to take into account the representation (4.7).

Returning to representation (4.15), we make use of the following observations. If $a: \bar{\Omega} \rightarrow \mathbb{R}$ is a function satisfying the properties (j)-(jv), then the equality

$$
u(x)=\frac{1}{(\sqrt{a(x)})_{x}}\left[(\sqrt{a(x)} u(x))_{x}-\sqrt{a(x)} u_{x}(x)\right]
$$

holds true for all $u \in H_{a, 0}^{1}(\Omega)$ and $x \in \bar{\Omega} \backslash\left\{x^{0}\right\}$. Therefore,

$$
\begin{aligned}
\|u\|_{L^{2}(\Omega)} & \leq\left\|\frac{1}{(\sqrt{a})_{x}}\right\|_{L^{\infty}(\Omega)}\left[\left\|(\sqrt{a} u)_{x}\right\|_{L^{2}(\Omega)}+\left\|\sqrt{a} u_{x}\right\|_{L^{2}(\Omega)}\right] \\
& \leq\left\|\frac{1}{(\sqrt{a})_{x}}\right\|_{L^{\infty}(\Omega)}\left[\|u\|_{V_{a}^{1}(\Omega)}+\|u\|_{H_{a}^{1}(\Omega)}\right] \\
& \left.\leq\left\|\frac{1}{(\sqrt{a})_{x}}\right\|_{L^{\infty}(\Omega)}\left(1+C_{H}\right)\right)\|u\|_{H_{a}^{1}(\Omega)} .
\end{aligned}
$$

However, in view of the properties $(\mathrm{j})-(\mathrm{jv})$, this estimate becomes consistent if only

$$
(\sqrt{a(x)})_{x}=\text { const in } \Omega .
$$

This motivates us to the following conclusion.

Proposition 4.2. Let $a: \bar{\Omega} \rightarrow \mathbb{R}$ be a weight function which is defined as follows

$$
a(x)=C_{*}^{2}\left(x-x^{0}\right)^{2}, \quad \forall x \in \Omega=(0,1),
$$

where $C_{*}>0$ is a given constant. Then

$$
\|u\|_{L^{2}(\Omega)} \leq \frac{1+C_{H}}{C_{*}}\|u\|_{H_{a}^{1}(\Omega)} \leq \frac{2 \sqrt{1+C_{*}}}{C_{*}}\|u\|_{H_{a}^{1}(\Omega)}, \quad \forall u \in H_{a, 0}^{1}(\Omega),
$$

and $\left(\int_{\Omega}\left(x-x^{0}\right)^{2} u_{x}^{2} d x\right)^{1 / 2}$ is an equivalent norm to the standard one in $H_{a, 0}^{1}(\Omega)$. 
In conclusion, we would like to emphasize the following fact: If the weight function $a(\cdot)$ satisfies properties $(\mathrm{j})-(\mathrm{jv})$, then the elements of the space $H_{a, 0}^{1}(\Omega)$ are not necessary continuous functions ( see Proposition 3.1 for comparison).

Example 4.1. Let $x^{0}=0.5$. Setting $a(x)=\left|x-x^{0}\right|^{4}$, we see that properties (j)-(jv) hold true. We define the following functions

$y(x)=\left\{\begin{array}{ll}\left|x-x^{0}\right|^{-\frac{1}{4}}-\left|x^{0}\right|^{-\frac{1}{4}}, & \text { if } x \in\left(0, x^{0}\right), \\ \left|x-x^{0}\right|^{\frac{1}{2}}, & \text { if } x \in\left(x^{0}, 1\right),\end{array} \quad u(x)=\sqrt{a(x)} y(x), \quad \forall x \in \Omega\right.$.

Then, in spite of the fact that the function $y: \Omega \rightarrow \mathbb{R}$ has a discontinuity of the second kind at $x^{0}=\frac{1}{2}$, the direct calculations show that $(\sqrt{a})_{x} \in L^{\infty}(\Omega)$, $u \in W_{a}(\Omega)$, and $y \in V_{a, 0}(\Omega)$. Hence, $y \in H_{a, 0}^{1}(\Omega)$ by Theorem 4.1. At the same time, $u(x)=\sqrt{a(x)} y(x)$ is the absolutely continuous function in $\Omega$.
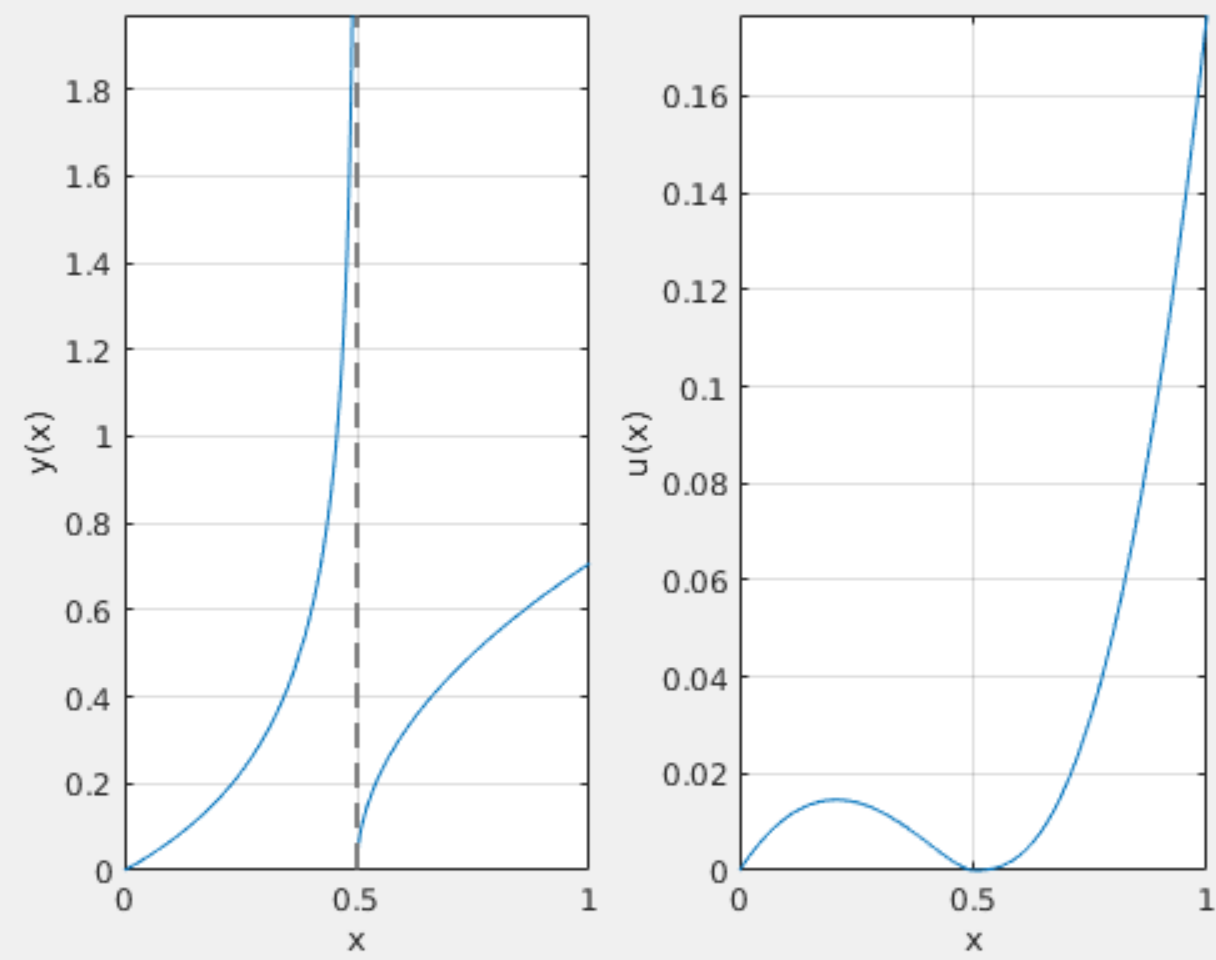

Fig. 4.1. Plots of functions $y(x)$ and $u(x)$ 


\section{Acknowledgment}

The authors would like to thank the DFG for the financial support of this research that was done in the framework of joint German-Ukrainian project "Sustainable Optimal Controls for Nonlinear Partial Differential Equations with Applications: Relaxation, Approximation and Optimality Conditions" under Grand 405372818.

\section{References}

1. F. Alabau-Boussouira, P. Cannarsa, G. Leugering, Control and stabilization of degenerate wave equations, SIAM J. Control Optim., 55 (3) (2017), 20522087.

2. F. Alabau-Boussouira, P. Cannarsa, G. Fragnelli, Carleman estimates for degenerate parabolic operators with applications to null controllability, J. Evol. Equ., 6 (2006), 161-204.

3. P. Cannarsa, P. Martinez, J. Vancostenoble, Carleman estimates for a class of degenerate parabolic operators, SIAM J. Control Optim., 47(2008), 1-19.

4. V. Chiadó Piat,F. Serra Cassano, Some remarks about the density of smooth functions in weighted Sobolev spaces, J. Convex Analysis, 1 (2) (1994), 135-142.

5. M. Colombo, A. Figaldi, Regularity results for very degenerate elliptic equations, J. Math. Pures Appl., 101(2014), 94-117.

6. P. Drabek, A. Kufner, F. Nicolosi, On the solvability of degenerate quasilinear elliptic equations of higher order, J. Differential Equations, 109(1994), 325-347.

7. M. GueYe, Exact boundary controllability of 1-D parabolic and hyperbolic degenerate equations, SIAM J. Control Optim., 52(2014), 2037-2054.

8. A. Greenleaf, Y. Kurylev, M. Lassas, G. Uhlmann, Cloaking devices, electromagnetic wormholes, and transformation optics, SIAM Rev., 51(2009), 3-33.

9. P.I. Kogut, G. Leugering, Optimal Control Problems for Partial Differential Equations on Reticulated Domains. Approximation and Asymptotic Analysis, Series: Systems and Control, Birkhäuser Verlag, Boston, 2011.

10. P. I. Kogut, G. Leugering, Optimal $L^{1}$-control in coefficients for Dirichlet elliptic problems: $W$-optimal solutions, Journal of Optimization Theory and Applications, 150(2)(2011), 205-232.

11. P. I. Kogut, G. Leugering, Optimal $L^{1}$-control in coefficients for Dirichlet elliptic problems: $H$-optimal solutions, Zeitschrift für Analysis und ihre Anwendungen, 31(1)(2012), 31-53.

12. P. I. Kogut, G. Leugering, Matrix-valued $L^{1}$-optimal controls in the coefficients of linear elliptic problems, Zeitschrift für Analysis und ihre Anwendungen, 32(4)(2013), 433-456.

13. G. Marinoschi, R. M. Mininni, S. Romanelli, An optimal control problem in coefficients for a strongly degenerate parabolic equation with interior degeneracy, J. Optim. Theory Appl, 173(2017), 56-77.

14. F. Santambrogio, V. Vespri, Continuity in two dimensions for a very degenerate elliptic equation, Nonlinear Anal., 73(2010), 3832-3841.

15. V. V. Zhikov, On Lavrentiev phenomenon, Russian J. Math. Phys. 3 (2) (1994), 249-269.

16. V. V. Zhikov, Weighted Sobolev spaces, : Mathematics, 189 (8) (1998), 27-58. 